\title{
Teaching model-based development methods for biomedical signal processing to sensitize and motivate for accuracy and product quality
}

\author{
Felderhoff, T. \\ Dortmund University of Applied Sciences and Arts \\ Institute for Information Technology \\ Dortmund, Germany \\ felderhoff@fh-dortmund.de
}

\begin{abstract}
In many applications it seems to be clear, what nowadays is possible through the evaluation of sensor signals or is represented as possible. Particularly the newest applications to smartphones, called Apps briefly, let us be astonished again and again. Periodical vibrations while keeping a smartphone in hand or the evaluation of a video when a finger is put on the camera, should enable conclusions about the user's heartbeat. Regardless of how you may refer to those examples, they point out quite clearly that by using modern sensor technology an observation of the physical constitution and the activity and movement of people in all circumstances of life appears as possible. In addition, an objective quality assessment is needed in order to justify the inevitable conclusions on constitutional fitness are at all useful.

The model-based development is used in the automotive industries for years under the aspect of a high quality. This development methodology is transferable to the filed of biomedical engineering. This paper shows how the model-based development is applicable particularly to biomedical signals. It is clarified at a first simple example how students can be sensitized to the necessary accuracy of signal processing and for the fulfilment of quality requirements apart from the pure development of processing and analysis algorithms. Thus, the added value of this development methodology is described clearly.
\end{abstract}

\section{Introduction}

The reference from theory to practice is a decisive factor in the education to a responsible engineer. The foundation of every education must be the teaching of the mathematical, physical and natural fundamental knowledge [1,2]. Based on this specialized advanced topics are offered which lead to a specialization of the students depending on their disposition.

In the field of information technology and communications such specializations are often divided into a hardware- or software-oriented direction. A hardware orientation includes for example the development of modern electronic circuits corresponding to the required components of analogue and digital processing units. A software-oriented approach often used such circuits to implement algorithms on the microprocessor of the digital processing unit. The algorithms will perform the desired tasks.

Because of the variability of the required competences, they are united mostly in more than one engineer. Team oriented work is common for engineers. For this, however, it is absolutely necessary that the interfaces are completely defined between the individual areas of expertise. Unfortunately, the requirements on these interfaces are not all really known in advance. Many specifications only arise during development. So this does not have any serious negative effects, a close dialogue between the engineers is required to react to such changes and their impact immediately. This is not an easy task.
Modern development tools support the engineers but just at this point. Time and cost reasons are not necessarily the decisive factors for the use of these development tools in the education of students. But at universities the workpackages of a project are executed by several students, not assigned to a project team at the same time. The fluctuation during a project is significantly larger at universities than in industrial enterprise. Therefore it is necessary to ensure that previously developed results are documented and subsequent work precisely can be based on these results.

\section{Model-based development}

The associated methodology is called model-based development. In the industry, primarily in the automotive industry, this methodology is pursued to

- provide a well documented and team-orientated work,

- customize and reuse subtotals of previous development work more easily,

- respond as flexible as possible to changes in the specification during the entire development process,

- reduce costs even with increased flexibility,

- have convenient test mechanisms by which the fulfilment of the specification can be shown and which ultimately lead to an increase in the quality of the product, and finally,

- submit partial results of certification, which point out the functionality and the correct compliance of specified standards. 
Due to other legal framework conditions, this development methodology is not directly transferable to the area of medical engineering either. Nevertheless, there are decisive steps enclosed, which could be useful applied to the development of technical medical or rehabilitation devices. In particular, efforts to reduce development time, i.e. from the idea to market, and ensuring high quality standards with the possibility of certification are key factors for a model-based development methodology in the field of medical technology.

model of product algorithm model of target system

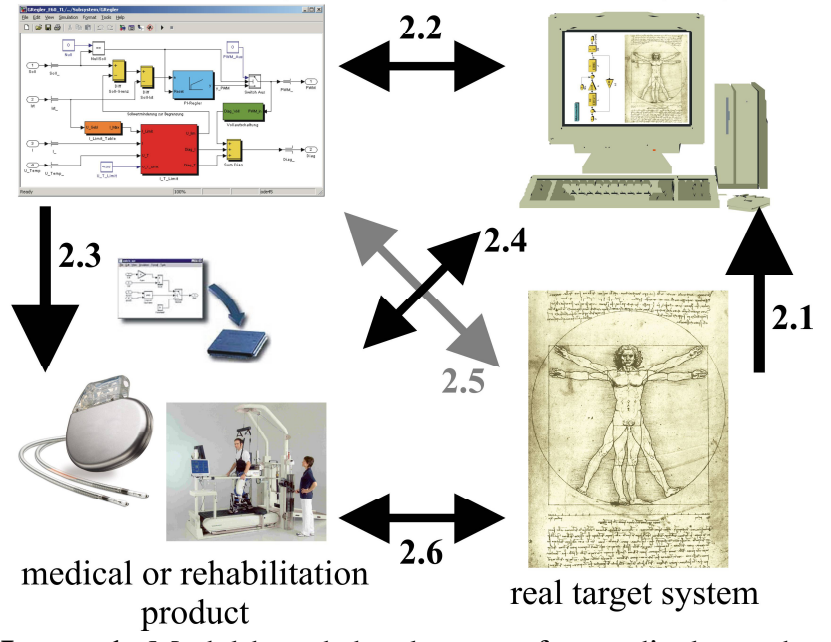

Image 1: Model-based development for medical or rehabilitation products

In the following essential steps of this development methodology are described. Image 1 illustrates the relationships between the single parts. For model-based development of medical or rehabilitation devices we will start modelling the real target system, i.e. the human (chapter 2.1). The design step from target system's model to the development of the product's algorithm is described in chapter 2.2. Automated code generation for the new product (chapter 2.3) and the essential test mechanism (chapter 2.4) show how to increase the quality of the product. Rapid prototyping (chapter 2.5) and the integration on the real target system (chapter 2.6) complete this brief description of modelbased development.

\subsection{Modelling}

Starting point is the desired real target system for which the medical or rehabilitation product is developed. To describe the mode of action of the product to be developed on a PC and its development tools as realistically as possible, the real target system has to be modelled, i.e. it has to be described by its mathematical an physical contexts [1$3]$. On the one hand this could be larger movements of the extremities, or on the other hand this also could be detailed mechanisms of the metabolism at the cellular level.

The level of detail which is required depends strongly on the particular tasks. A model-based development starting on such a model is only as good as the underlying real target system's model. The requirements to the model can be extended gradually and adapted to the needs. The objective of the engineer has to be that the real target system, e.g. a human being, is described as realistic and exact as necessary. The challenge here is that the real target system, such as a human, is just not a technical system, such as an automobile. Thus, it requires a considerably more complex kind of modelling containing deterministic and stochastic components $[3,4]$. Additionally, the individuality of each human being has to be taken into account.

This can be achieved by using an appropriately parameterizable modelling or by using real recorded data containing the diversity of these humans.

\subsection{Algorithm development and software- in-the-loop test}

Now an adequately accurate model is available in the development tool. In this software environment the development of the product's software is worked on now. The product's main processing units are typically microprocessors. The development of algorithms for these microprocessors represents the software-oriented part of the product development.

A significant advantage is that the operation of the algorithm can be tested in the sense of software-in-the-loop tests and it can be verified against the model of the real target system. On the one hand the functionality of the product to be developed is comprehensively tested with sufficient accuracy of the real target system's model or with sufficient diversity of real data, respectively. On the other hand these test mechanisms are independent of already developed hardware for the product. Because of this potential parallelism of software and hardware development, an overall reduction of the complete development time arises for the product.

\subsection{Automated code-generation}

Moreover, the characteristics and the performance of the microprocessor, e.g. data type format and word length or the arithmetic of the microprocessor's processing unit, also can be taken into account to get an exact simulation of the signal processing algorithm on bit level. Using such model-based development tools, this leads to the automated generation of code for the microprocessors.

Transmission or implementation errors in manually programming the microprocessor will be avoided this way. A certification of software by appropriately extensive tests is then transferable directly to the implemented code. Beside a lower error rate this approach increases the quality of product, and results in an effective software development. The advantages of this automatism are bought by the overhead in automated code-generation.

\subsection{Hardware-in-the-loop test}

With this result now it is possible to implement the developed software on the in parallel developed hardware of the product. In principle, the use of the new product on the real target system, i.e. on human beings, is generally possible. The advantages of a hardware-in-the-loop test have to be 
considered here, because they are able to minimize the potential risks again significantly. It is easy to test the actually finished product against the model of the real target system. So at the model it is checked that all requirements of the product are met and that it has the desired functionality and safety.

Furthermore such critical tests can be carried out, where extremely improbable reactions of the real target system are described in its model to observe the resulting reaction of the product in such cases. Moreover, consequences of errors on the real target system can be simulated and estimated observing the model's reactions. Here again, it becomes clear that the relevance of such statements depend on the accuracy and realistic description of the model.

\subsection{Rapid Prototyping}

Depending on the risks, in principle a verification of the developed software against the real target system is possible. But be aware that rapid prototyping in the field of medical technology has to meet the same strict requirements as the medical product or device itself.

\subsection{Integration}

The launch of the new or improved product stands at the end of each development. Since all development steps were verified and tested against each other during the complete developmental phase, a product results of high quality.

Safety requirements can be formulated exactly as the requirements to hardware or interfaces. The compliance with these requirements in turn leads to appropriate tests so that the product will pass the clinical trails. Due to the transparency of each development step, a certification of developed partial solutions seems to be possible.

\section{$3 \quad$ Biomedical and kinematic data- logger}

To be able to develop such models now, the reality must be adequately exact observed and analyzed. For this the most important base are measured physiological and kinematic data which characterize the behaviour and reaction of the real target system corresponding to the product's functionality. High-performance data loggers are required for multi-channel data acquisition and recording.

\subsection{Requirements}

The simultaneous acquisition of as many as possible or necessary data is desirable in high signal quality and high information content. This usually results in data words having accordingly many bits and a great sampling frequency. If a multi-channel data acquisition takes place on this basis then huge amounts of data have to be stored, managed and also analyzed.

However to be able to formulate concrete statements concerning a minimum of these requirements it is necessary to collect such high quality data sets during the initial phase of development. An analysis of cause and effect requires a time comparable map of these data, so that a time stamp for each sampled data seems to make sense.

Usually the data will be collected mobile. The data acquisition system must take this use into account. Thus, an energy efficient realization moves into the focus and the system has to ensure long term data collections.

Neither the data acquisition moreover should be restricting natural movements or activities nor it should be obvious to the test persons while data collecting.

An interaction between data collection system and test persons must be possible. But the preparation of information has to fit the needs of the test persons and not the developer's wishes.

\subsection{Hardware concept}

A possible and very flexible hardware concept is represented in image 2 . There a field programmable gate array (FPGA) is taken into account for parallel data acquisition. The data collection is possible using different sensors which either are connected to the data acquisition system directly by cables, or the data are transmit over a wireless connection [5]. For example, it would have to be decided using the requirements, whether an acceleration sensor attached to the foot transmit its data by wire or wireless.

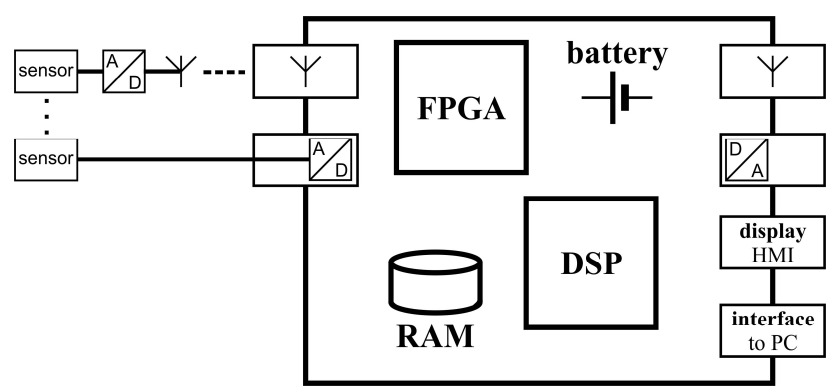

Image 2: Hardware concept

For a direct processing of collected signals it would be possible to use the digital signal processing (DSP) unit within some high-performance FPGAs. But using a separate DSP this hardware concept obtains greater flexibility for applications.

In mobile applications a fast and robust data acquisition and signal processing, respectively, is of great importance in the development of such a data logger. The field of applications depends on the kind of used sensors.

\subsection{Software concept}

The software concept depends on the kind of collected data and signal processing, which can go from a reduction of the amount of data by compression about the extraction and analysis of signal properties up to a control of actuators $[3,4]$.

The flexibility of different software on specific hardware is well-known by, e.g., personal computers. The flexibility of this data logger is therefore determined by the properties and performance of the hardware, particularly the FPGA and DSP. 


\subsection{Human machine interface}

The human machine interface (HMI) is a key factor for the success of a product. The more customized and more personalized the HMI design is the better the customer gets with the product.

This gets clear at this data logger or at any medical device if different customers are considered. Medical stuff or doctors have other expectations to the processed content and the influence on patients than a patient himself. A patient may only need to know whether everything is all right or whether he must contact to a medical facility.

\section{$4 \quad$ First feasibility study}

It is also an advantage of model-based development that with progressive knowledge gained this can lead to a concretization of the specification. Thus, an ambitious target can be pursued by stepwise carrying out improvements from a first feasibility study up to an improvement and expansion of the demonstrator.

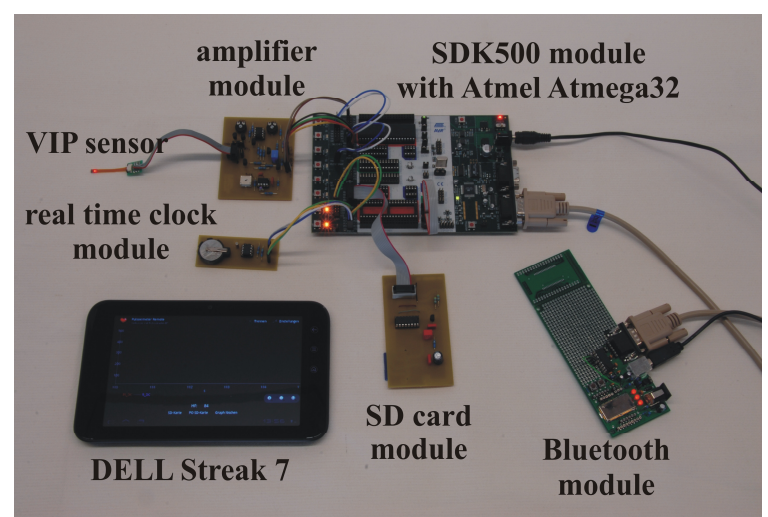

Image 3: First feasibility study

The first feasibility study, shown in image 3, permits the collection of pulse oximetry signal using the VIP-Sensor [6] in a simple way. An Atmel ATmega32 microcontroller provides the storage of collected data on a SD-card without further processing. For real time operation and without loss of any sample this bottleneck reduces the sampling frequency to $285 \mathrm{~Hz}$ whereas $1 \mathrm{kHz}$ is desired [7]. Thanks to the used real time clock, which generates time stamps for each sample, it was possible to detect the loss of some samples at higher sampling frequency [8].

The stored signal can be processed by the SD card at a PC. A wireless data exchange is possible via Bluetooth, too. This is valid for both, directly sampled data or stored data. A first front-end of a HMI is developed for a tablet-PC [9] running Android 3.2 operating system. Due to the limited performance of the microcontroller on the data logger, the calculation of the heart rate is put on the tablet-PC. This has currently the consequence that no extracted data are transmitted but the whole amount of sampled data [8].

This first study is no more than the demonstration of the principle feasibility. Furthermore it has got obvious because of the restricted performance that you have to pay attention on the performance and quality of each component.

\section{Conclusion}

Objective criteria allow evaluating the effectiveness of new medical or rehabilitation products. Primarily characteristic values are included with an appropriate relevance to the specific functionality of the product.

Such collected data play a crucial role in model-based development because the modelling is carried out with them. Different values have quite different relevance and different information content for a concrete task, respectively. The knowledge of the information content is essential for an effective, i.e. target-oriented data collection. Signal characteristics can only be extracted from the values if they are included in the collected data at all. During the development phase these characteristics are not necessarily known a priori. For the analysis of signals and the search for specific properties significantly more data are required than afterwards may be used in the final product.

A special attention should be paid to this data acquisition. Information not included cannot be reconstructed later. Furthermore the potential of signal processing of biomedical signals can be represented particularly well if the signals to be processed are of high quality. Not only the pure data acquisition and subsequently analysis by a doctor is possible but new data logger products may perform potentially important analysis and propose diagnosis in real time. The doctor will fast and reliably be provided with important information in the field of diagnosis through the continuous monitoring of patients in real life environment.

\section{References}

[1] Northrop, R.B., Signals and Systems Analysis in Biomedical Engineering, CRC Press, 2003

[2] Baura, G.D., System Theory and Practical Applications of Biomedical Signals, IEEE Press, 2002

[3] Bronzino, J.D., The Biomedical Engineering Handbook, CRC Press IEEE Press, 1995

[4] Husar, P., Biosignalverarbeitung, Springer Verlag, 2010

[5] Wang, P. and Liu, Q., Biomedical Sensors and Measurement, Springer Verlag, 2011

[6] CiS Forschungsinstitut für Mikrosensorik und Photovoltaik GmbH, Microoptical Sensor VIP preliminary datasheet, 2011

[7] Böhmer, A., Konzeptionierung eines mobile Datenloggers zur Aufzeichnung von Vitalparametern und prototypische Entwicklung unter Verwendung eines ATmega32-Mikrocontrollers und eines VIP-Sensors, Bachelor-Thesis, Dortmund University of Applied Sciences and Arts, 2011

[8] Regner, P., Entwicklung einer Mensch-MaschineSchnittstelle zur Anzeige und Auswertung medizinischer Messdaten auf einem Tablet-Computer unter Android, Bachelor-Thesis, Dortmund University of Applied Sciences and Arts, 2011

[9] Dell Inc., Dell Streak 7 Android Tablet PC, Version 2012 